\title{
Rice Starch-Based Biodegradable Films: Properties Enhancement
}

\author{
Thawien Wittaya
}

Additional information is available at the end of the chapter

http://dx.doi.org/10.5772/47751

\section{Introduction}

The claims of environmental protection and the lack of petroleum resources provide a new opportunity for developing plastic materials derived from biopolymer resources. Starch is one of the most studied and promising raw materials for the production of biodegradable plastics, because starch is quite cheap, abundant, biodegradable and edible. Starch consists of two major types of molecules, primarily linear amylose and highly branched amylopectin. Normal starch consists of about $75 \%$ amylopectin and $25 \%$ amylose; waxy starches consist of mainly amylopectin and 0-8\% amylose; and high-amylose starches consist of $40-70 \%$ amylose. Amylose is composing of D-glucose molecules, which are linked in a $\alpha-1,4$ conformation. The glucose monomers therefore form a linear straight chain polymer. Amylose is the key component involved in water absorption, swelling and gelation of starch in food and processing of material (Hoseney, 1986).

Amylopectin is the major component of most starches, and consists of a large number of shorter chains that are bound together at their reducing end side by a $\alpha-1,6$ linkage (Hoseney, 1986). Amylopectin is therefore highly branched as the $\alpha-1,4$ linear chains are punctuated with the $\alpha-1,6$ linkages. The $\alpha-1,6$ constitute about $5 \%$ of the structure of amylopectin and gives rise to branching. The amylopectin molecule is much larger than the amylose molecule. Minor components, such as lipids, phospholipids, and phosphate monoester derivatives, are found in starch and have profound effects on the properties of starch. Lipids and phospholipids are found in cereal starches. Normal cereal starches contain up to $1 \%$ lipids, and the level of lipid content is proportional to the amylose content of the normal starch (Morrison, 1995). Starches of different botanical origins consist of different species of lipids. For example, normal maize starch consists of mainly free fatty acids, glycerides, and little phospholipids; normal rice starch contains substantial amount of phospholipids and some free fatty acids; and wheat, barley, rye, and triticale starches consist 
exclusively of phospholipids (Kasemsuwan \& Jane, 1996; Morrison, 1995). Cereal waxy starches contain few lipids, whereas high-amylose starches contain substantially more lipids. Root and tuber starches contain very little lipids and no detectable phospholipids (McPherson \& Jane, 1999).

Rice is the most widely consumed basic food in the world. Each year over 500 million tons of rice are harvested, providing sustenance to many countries and people throughout the world. The unique properties of rice starches are found in its many varieties. Due to different climates, soil characteristics and cultures, over 240,000 registered varieties of rice exist in the world. These varieties lead to a wide range of rice starches with many different characteristics including: different onset gelatinization temperatures; textures; processing stability; and viscosities. Rice starch with high amylose is an attractive raw materials for use as barriers in packaging materials. They have been used to produce biodegradable films to partially or entirely replace plastic polymers because of the low cost and renewability, as well as possessing good mechanical properties. However, compared to the common thermoplastics, biodegradable rice starch products still reveal many disadvantages. These include low mechanical properties and lack of efficient barrier against high polarity compounds. The disadvantages are mainly attributed to the highly hydrophilic character of rice starch polymers. To cope with these problems while preserving the biodegradability of the materials, the improvement of rice starch film properties has been investigated to meet suitable applications. This chapter provides details of rice starch films, and the formation and factors affecting rice starch film properties. It also deals with improving rice starch film properties with various methods such as using chemical treatments, physical treatments, combination with other biodegradable materials and fiber reinforcement.

\section{Formation of rice starch-based biodegradable films}

The formation of rice starch films requires the use of at least one constituent capable of forming a matrix with adequate continuity and cohesion. Generally, this is composed of polymers or compounds which, under the preparation conditions, are used to form continuous crystalline or amorphous products. In the case of coatings for which the films' system can be applied directly to the product, two forces are relevant: that between the molecules of the coating material (cohesion) and that between the coating and the support structure (adhesion). The degree of cohesion produces the barrier and mechanical properties of the film. High structural cohesion is manifested by a reduction in flexibility, porosity, and permeability to gases and solutes (Banker, 1966). The degree of cohesion depends on the chemical structure of the film material, the presence of plasticizing and cross-linking agents, the nature of the solvent used and its dilution, the method of application, the procedure used for removal of the solvent, and the final thickness of the film.

The highest cohesion is generally obtained for ordered polar polymers with long chains which were precipitated in crystalline form. The preparation for average dilution which constitutes a compromise between the salvation and extension of polymer molecules and good initial viscosity is preferable (Baker, 1966). The cohesion of films generally increases in 
proportion to their thickness up to a threshold beyond which it remains constant. The speed of evaporation of the solvent and/or excessive temperature may be manifested by inadequate cohesion on account of the premature immobilization of the polymer molecular. Regarding the adhesion of the coatings to the foodstuff, this is generally facilitated by hot application.

The formulation of films base on starch may require one of the following methods. (1) Casting, which is a process consisting of drying a solution or a gel is a simple method for producing films with controlled thickness. This technique is useful to mimic some industrial processes for forming free-standing starch films as is the case for dip-molding. In this method, used for food coatings as well as for non-food applications, the gelled state is usually preferred to set hot solutions on a surface upon cooling. (2) Extrusion or the thermo pressing process is a process used to create objects of a fixed cross sectional profile. A material is pushed or drawn through a die of the desired cross section. The two main advantages of this process over other manufacturing processes are its ability to create very complex cross sections and work materials that are brittle, because the material only encounters compressive and shear stresses. It also gives finished parts an excellent surface finish. Next is (3) Electrohydrodynamic atomization (EHDA), referred to as electrostatic atomization or electro-spraying. This is a process in which a liquid is forced through a capillary and a potential difference of the order of kilo volts is applied between the capillary and the collection electrode (Pereta \& Edirisinghe, 2006). EHDA can conduct in various modes but the stable cone-jet mode is the most desirable as it provides near-monodispersed droplets of a few micrometers in size. The droplets size can be controlled by the flow rate and applied as appropriate (Clopeau \& Prunet-Foch, 1990).

\section{Factor affecting rice starch-based biodegradable films}

\subsection{Amylose and amylopectin content}

Starch consists of two polysaccharides, the essentially linear amylose, and the branched amylopectin (Manners, 1989). The pure amylose structure is very stable, with strong molecular orientation, forming films denser and stronger than amylopectin films (Lourdin et al., 1995). The ability of amylose to produce self supporting films has been known for a long time and this is attributed to the ability of its linear chains to interact by hydrogen bonds to a higher extent than the branched amylopectin chains. Amylopectin films, on the other hand, are rather frail due to the higher degree of entanglement caused by the extensive branching and the short average chain length (Rindlav-Westling et al., 1998). RindlavWestling et al. (2002) prepared films from potato starch, amylose, and amylopectin and blends by solution casting. Results showed that amylose films had a relative crystallinity of about $30 \%$ whereas amylopectin films were entirely amorphous. The blending of amylose and amylopectin resulted in films with a considerably higher degree of crystallinity than could be predicted. This is explained by co-crystallization between amylose and amylopectin and possibly by crystallization of amylopectin. The crystallized material gave rise to an endothermic detected with differential scanning calorimetry. The enthalpy and 
peak temperature of the transition also increased as the amylose content decreased. When the amylose proportion in the blends was low, separate phases of amylose and amylopectin were observed by light microscopy. At higher amylose proportions, however, the phase separation was apparently prevented by amylose gelation and the formation of continuous amylose network. Addition, the amylose network in the films, observed with transmission electron microscopy, consisted of stiff strands and open pores and became less visible as the amylose proportion decreased.

Alves et al. (2007) studied the effect of amylose enrichment on cassava starch films properties. This study showed the mechanical and barrier properties of cassava films were influenced by the amylose contents. The amylose enrichment originated stronger films and this could be explained because during drying of film-forming solutions, water evaporates, allowing the formation of starch network. During this stage the proximity of starch chains induced by higher amylose contents could facilitate the formation of matrix with more polymer content per area.

Ming et al. (2011) characterized the biodegradable films from corn starch with different amylose content. They concluded that amylose content had significantly affected the mechanical and thermal properties of the biodegradable starch-based films. The high amylose starch films exhibited better mechanical properties, such as higher modulus and tensile strength, and very high impact strength. The reasons for this include not only the easy entanglement of long linear amylose chains, but also the retained granular structure in high amylose films, which may act as self reinforcement.

Muscat et al. (2012) studied the effect of low and high amylose starches on film forming behavior. They found that, films with high amylose content showed higher glass transition temperature, tensile strength and modulus of elasticity values and lower elongation values than low amylose starch films. There was an increase in thermal and mechanical properties of high amylose starch films. This could be because of what happens when the drying of film-forming solutions, water evaporates, and allowing the formation of starch network takes place. During this stage, the proximity of starch chains induced by higher amylose contents could facilitate the formation of a matrix with more polymer content per area as well (Alves, et al., 2007).

\subsection{Type and content of plasticizers}

Native starch films are brittle compared with synthetic polymers such as polyethylene, and technically need to be plasticized. A plasticizer is substance that is incorporated into rigid materials to increase its flexibility, workability, and dispensability. By reducing the grass transition temperature and increasing chain lubricity, plasticizers could also improve processing and extrusion characteristics. They could also reduce the minimum required processing temperature, reduce the plastic's hardness and improve low temperature flexibility.

Generally, two types of plasticizers are distinguished. Internal plasticization is a result of modifications to the chemical structure of polymer. External plasticization is obtained by 
adding an agent which modifies the structure and energy within the tree-dimensional arrangement of the film polymer (Banker, 1966). It is the second method which, on the basis of the type of materials and the technology used, is mainly used for biodegradable packaging. The addition of a plasticizer to a film produces a film which is less likely to break and is more flexible and stronger.

Basically, the plasticizers should be generally compatible to the structure of the polymer that they plasticize and the permeability be present within the solvent-polymer system and under the conditions used. To be compatible, it must be compatible with the polymer, which results in the inter-molecular reactions. It is important to note that the formulation of the whole film system (polymer, solvent, plasticizer, and other additives) has a direct effect on the nature and characteristics of the film produced. As a result, the polymer and the plasticizer must not only be compatible, but must also have similar solubility in the solvent used. A soluble plasticizer will generally be sought for the development of soluble coating and an insoluble plasticizer (or a dispersible one) for an insoluble coating or for a slow solubilization.

The permanence of a plasticizer is also of prime importance since this influences the physical and mechanical stability of the film. The plasticizer should not be volatile (or not only very slightly volatile) and its degree of retention by the film should be high. Other properties, such as its chemical stability, hygroscopicity, color, flavor, and so on, are also more or less important depending on the type of film under consideration. In addition, the content of plasticizer necessarily varies from $10-60 \%$ (dry basis) according to the nature and type of film and the method of application. The plasticizers that are most usually used in the field of rice starch films are mono-, di-, and oligosaccharides, polyols and lipids and its derivatives. The molecular size, configuration and total number of functional groups of the plasticizer as well as its compatibility with the polymer, could affect the interactions between the plasticizer and the polymer (Yang \& Paulson, 2000).

Bourtoom \& Chinnan (2008) determined plasticizer effect on the properties of biodegradable blend film from rice starch-chitosan. The results of these studies demonstrated that sorbitol plasticized films provided the films with highest mechanical resistance, but the poorest film flexibility. In contrast, glycerol and polyethylene glycol plasticized films exhibited flexible structure; however, the mechanical resistance was low, while inversely affecting the water vapor permeability.

The effectiveness of glycerol in biodegradable blend films from rice starch-chitosan is most likely due to its small size which allows it to be more readily inserted between the polymer chains. It consequently exerts more influence on the mechanical properties than the larger polyethylene glycol molecule. In addition, at an equal percentage of concentration, the total number of glycerol molecules in the film solution is greater than that of the higher molecular weight polyethylene glycol. Therefore glycerol has more functional groups $(-\mathrm{OH})$ than polyethylene glycol which should promote the plasticizer-polymers interactions in the films. As a result of the glycerol, plasticized films provided the films with higher water vapor permeability than polyethylene glycol, and sorbitol should be the result of the high 
hydrophillicity of the glycerol molecule, which is favorable to the adsorption of water molecules and could also contribute to the increase in the film water vapor permeability. In addition, at high glycerol concentration, glycerol could cluster with itself to open the polymer structure, enhancing the permeability of the film to moisture (Lieberman \& Gilbert, 1973). An increase in inter chain spacing due to the inclusion of glycerol molecules between the polymer chain may promote water vapor diffusivity through the film and hence accelerate the water vapor transmission (Yang \& Paulson, 2000).

Dai et al. (2010) reported that type and content of plasticizer affected the properties of corn starch films. Increasing the plasticizer content resulted in increasing water vapor permeability of the resulting film. These results would be related to structural modifications of the starch network brought about by the plasticizer concomitant with the hydrophilic character of plasticizer, which favored the absorption and desorption of water molecules. Plasticizers reduced intra- and inter-molecular forces in starch. In addition, plasticizers could extend, dilute and soften the structure effectively; then the starch chain mobility would be increased.

\subsection{Type and content of lipids}

Biodegradable starch films generally provide a good barrier against oxygen at low and intermediate relative humidity, and have good mechanical properties, but their barrier against water vapor is poor due to their hydrophilic nature (Kester \& Fennema, 1986). In contrast, films prepared with lipid materials have good water vapor barrier properties, but are usually opaque and relatively inflexible. Lipid compounds commonly used for the preparation of lipid-based biodegradable films include neutral lipids, fatty acids, waxes, and resins (Kester \& Fennema, 1986; Hernandez, 1994). The way to obtain a better water vapor barrier in starch films is to produce a composite film by adding hydrophobic components such as lipid and wax materials. A composite starch-lipid film is particularly desirable, since it has acceptable structural integrity imparted by the starch materials and good water vapor barrier properties contributed by the lipid materials (Greener \& Fennema, 1989). The efficiency of the lipid materials in composite films depends on content and the nature of the lipid used such as structure, chemical arrangement, crystal type, shape, size, distribution of lipids, nature of barrier components, the film structure (including homogeneity, emulsion, multilayer.), and thermodynamics such as temperature, vapor pressure, or the physical state of water in contact with the films (Rhim \& Shellhammer, 2005).

Haggenmaier \& Shaw (1990) tested the effect of stearic acid concentration on the water vapor permeability of hydroxypropyl methylcellulose composite films. It was found that the water vapor permeability of the composite films decreased about 300 times with the addition of $40-50 \%$ of stearic acid. However, excessive levels of lipid materials result in the film becoming brittle. Yang \& Paulson (2000) investigated gellan/lipid composite films through emulsification and determining the effect of lipid (beeswax and 1:1 blend of stearicpalmitic acids) on the moisture barrier, and mechanical and optical properties of the films. The results depicted that the addition of the lipids to gellan films significantly improved the 
water vapor permeability $(\mathrm{p}<0.05)$, but lowered the mechanical properties and caused the films become opaque. Beeswax was more effective than stearic-palmitic acids in reducing the water vapor permeability and films with beeswax showed better mechanical properties overall than those with stearic-palmitic acids. Srinivasa et al. (2007) studied the effect of fatty acids (stearic and palmitic acids) on the mechanical and permeability characteristics of chitosan films. No considerable differences in water vapor permeability were observed in fatty acid blend films.

Bourtoom \& Chinnan (2009) investigated the effect of lipid types (oleic acid, palm oil, and margarine) and content of lipids on water vapor permeability, tensile strength, percentage of elongation at break, and structure of rice starch-chitosan composite film. Tensile strength and water vapor permeability of rice starch-chitosan composite film decreased with the addition of lipids, whereas elongation at break increased in these films. In general, lipid films lack the structural integrity of polysaccharide films. Therefore, incorporation of lipids into hydrophilic polysaccharide films in an effort to decrease their water vapor permeability can negatively affect film strength as expressed by tensile strength measurements. The increase in the lipid content causes a partial replacement of lipids in the film matrix. The interactions between non-polar lipid molecules and between the polar polymer and nonpolar lipid molecules are believed to be much lower than those between the polar polymer molecules. In addition, rice starch-chitosan films added with oleic acid provided the films with smoother surface and higher values of tensile strength and elongation at break but lower water vapor permeability than with margarine and palm oil, respectively. The differences in mechanical and barrier properties between these films could be related to their physical state, structure, and chemical nature of the lipids.

\subsection{Relative humidity}

Relative humidity is a term used to describe the amount of water vapor in a mixture of air and water vapor. It is defined as the ratio of the partial pressure of water vapor in the airwater mixture to the saturated vapor pressure of water at those conditions. The relative humidity of air depends on the temperature and pressure of the system. Relative humidity is often used instead of absolute humidity in situations where the rate of water evaporation is important, as it takes into account the variation in saturated vapor or pressure. Biodegradable starch films generally provide a good barrier against oxygen at low and intermediate relative humidity, and have good mechanical properties, but their barrier against water vapor is poor due to their hydrophilic nature (Kester \& Fennema, 1986). When starch films are exposed during storage time in certain environmental conditions, it is possible to obtain both physical and mechanical changes in their nature. Physical changes may be those such as polymer recrystallization (the retrogradation) and those due to the migration of low molecular weight components, such as plasticizers or water contained in film formulation.

This migration of additives can be considered the most important cause of physical instability of starch films. In order to diminish film fragility and increase film flexibility and 
manageability, plasticizers are added into film formulation (Guilbert, 1986). Water also acts as a plasticizer in hydrophilic films; the plasticizing effect of water is based on the weakening of hydrogen bonds and the dipole-dipole intra and intermolecular interactions due to shielding of these attracting forces by the water molecules. As a consequence, free volume increases which affects their mechanical properties. Stading et al (2001) studied the effect of relative humidity on amylose and amylopectin film properties. They found that when the relative humidity of surrounding films increase this yielded increasing water content and oxygen permeability. However, the storage modulus and glass transition temperature $(\mathrm{Tg})$ showed an inverse effect. The change in surrounding relative humidity affected the water content of the films. When the water content in the films increase this provides an increasing mobility of molecule in the network allows swelling with resulting heterogeneous network structure. Hence, sharply decreased storage modulus and glass transition temperature and increased the oxygen permeability of the resulting films.

Masclaux et al. (2010) reported that relative humidity affected the properties of starch nanocomposite films. They found that at high relative humidity, the water diffusion rate showed higher in the starch nanocomposite films. According to these results, it seemed that it was more water sorption and diffusion in starch matrix due to its initially high swelling capacity and high chain mobility. Beside, the results demonstrated that the oxygen permeability coefficient slightly increased in the range of relative humidity between 30 to $45 \%$ and greatly increased at higher relative humidity.

\section{Properties enhancement of rice starch-based biodegradable films}

\subsection{Cross-linking}

Cross-linking means that the polymer molecules are interconnected by some sort of bonding. The bonding can be covalent, ionic, or it can result from intermolecular forces such as hydrogen bonding. Cross-linking is used in both synthetic polymer chemistry and in the biological sciences. Although the term is used to refer to the "linking of polymer chains" for both sciences, the extent of cross-linking and the specifics of the cross-linking agents vary. Cross-linking can be accomplished chemically or by irradiation. Cross-linking is a key technique for modifying the properties of starches and can be achieved by adding intra- and inter-molecular bonds at random locations in the starch granules (Figure 1) (Singh et al., 2007). Cross-linking tends to limit the interaction of starch with water and provides a structural integrity of starch-based biodegradable materials during exposure to pressure and moisture (El-Tahlawy et al., 2007). Starch cross-linking is normally performed by treating starches (semi-dry or slurry) with reagents capable of forming either ether or ester linkages between hydroxyl $(-\mathrm{OH})$ groups on starch molecules. Poly-functional chemicals such as phosphorus oxychloride $\left(\mathrm{POCl}_{3}\right)$, sodium trimetaphosphate (STMP), sodium tripolyphosphate (STPP), epichlorohydrin (EPI), amixture of adipic and acetic anhydrides, and a mixture of succinic anhydride and vinyl acetate have been commonly used to crosslink starches (Singh et al., 2007). 
The type of cross-linking agent largely determines the change in functional properties of the treated starches. Starch phosphates may be grouped into two classes: monostarch phosphates and distarch phosphates (cross-linked starches). In general, monostarch phosphates (monoesters) can have a higher degree of substitution than distarch phosphates (diesters) (Singh et al., 2007). Singh et al. (2007) stated that a combination of substitution and cross-linking can provide stability against acid, thermal, and mechanical degradation of starch and delay retrogradation during storage. The mixture of STMP/STPP was a combination of monostarch phosphates (STPP) and distarch phosphates (STMP). STMP is reported to be an efficient cross-linking agent at high temperature with semi-dry starch and at warm temperature with hydrated starch in aqueous slurry (Kerr and Cleveland, 1962). EPI has poor solubility in water and partly decomposes to glycerol, thus water-soluble cross-linking agents such as STMP are preferred. $\mathrm{POCl}_{3}$ is an efficient cross-linking agent in aqueous slurry at $\mathrm{pH}>11$ in the presence of a neutral salt.

The biodegradable starch film prepared from cross-linked starch provides improved mechanical properties; improved abrasion/cut through; resistance to stress cracking; improved high temperature mechanicals; superior over load characteristics and decrease in flexibility (Rutledge et al., 1974). However, the degree of improvement depends on the type and content of cross- linking agent. Besides, the structure and molecular weight of the starch also affect the intermolecular interactions between molecules. Many researches have been done on the improvement of starch film by using cross linking agent. Kim \& Lee (2002) reported that the mechanical properties of starch films prepared with cross-linked corn starch show higher than native corn starch. In addition, Khan et al. (2006) showed that crosslinked sago starch/PVA blend films had mechanical properties higher than native sago starch/PVA blend films. The increase in mechanical properties is due to the increase of cross-linking density. The cross-linking agents react with the - $\mathrm{OH}$ groups present in starch and make ether linkages with the available hydroxyl groups. This helps to increase the mechanical properties (Das et al., 2010). This is because cross-linking reinforces the structure of starch granules and limits water absorption of starch thereby restricting the mobility of the starch chain in the amorphous region (Manoi \& Rizvi, 2010). According to the results of Das et al. (2010) the moisture absorption was considerably decreased in the a cross-linked starch/poly(vinyl alcohol) (PVA) blend films.

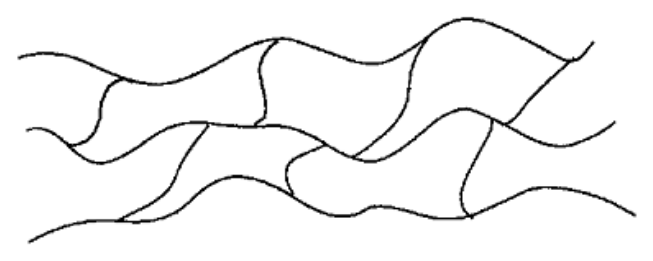

Cross-linking

Figure 1. Schematic of cross-linked polymer. Source: Smiley \& Jackson (2002) 


\subsection{Irradiation treatment}

Radioactive treatment, in solid or liquid states such as UV irradiation, gamma irradiation or electron beam have been extensively used to modify starch especially in the grafting process. Radiation processing technology is technically used to improve the properties of starch because of its tendency to undergo the chemical reaction between the polymer molecules under irradiation (Gehring, 2000). Irradiation can reduce sizes of starch molecules. Starch molecules can be broken down by free radicals formed from irradiation which can cause interruption in the molecular bonding structuring of the starch (Woods \& Pikaev, 1994).

\subsubsection{UV irradiation treatment}

Ultraviolet (UV) irradiation as a physical, cost effective, non-thermal, and environmentalfriendly technology has received increasing attention during recent years. During this time it has been successfully applied for preservation and decontamination of food products (Bintsis et al., 2000). UV irradiation has been used in medical and pharmaceutical research to crosslink collagen and gelatin films (Bhat \& Karim, 2009). UV irradiation requires the presence in the biological medium of certain substances known as photo-sensitizers which induce the changes in the biological substrate after absorbing appropriate radiation (Spielmann et al., 1994). The most efficient photosensitizers, sodium benzoate, are known to be photolysed by UV irradiation (Ghosp \& Gangopadhay, 2000).

The properties of biodegradable starch films could be improved by using UV irradiation depending on the intensity, treatment time and content of the photosensitizers. Some researches have been working on the improvement of starch based film by UV irradiation. Delville et al. (2002) studied a new family of cross-linked starches in the solid state by UV irradiation. The original photosensitizers used were water soluble and members of the benzoic acid family. They were able to cross-link starch even at concentrations as low as $0.1 \%$. The mechanical properties showed increases in the cross-linked samples. Follain et al. (2005) studied the impact of the addition of poly vinyl alcohol and photo cross-linking on starch based materials' mechanical properties. The mechanical properties of starch/PVA blends, with or without cross-linking, have been analyzed in order to study how the increase in the linear and branching chains can improve the material's performances. As a result, the mechanical properties of the films obtained were enhanced for both casting and extrusion processes when photo cross-linking was applied.

Khan et al. (2006) prepared and characterized ultra violet (UV) radiation cured biodegradable films of sago starch/PVA blend. Polymer films of sago starch/polyvinyl alcohol (PVA) were prepared by casting and curing under UV radiation. They found that UV irradiation could promote the degree of cross-linking of sago starch/PVA blend film. The tensile strength values of the films produced increased with UV radiation doses up to a certain limit and then decreased. According to these results, the cross-linking reaction between starch/PVA molecules may be induced while the UV light penetrated into 
starch/PVA film. The cross-linking reaction occurs through a radical mechanism; the sensitizer (sodium benzoate) is excited or decomposed to produce radicals upon irradiation with UV light (Figure 2). This radical reacted with starch/PVA molecule as a cross-linking reaction to increase the tensile strength of UV treated starch/PVA films . However, too strong a photo-sensitizer would inhibit the UV penetration and decrease the hydrogen abstraction (Delville et al., 2002). When the sensitizer was accessed the tensile strength was decreased. Kumar \& Singh (2008) improved the properties of starch biocomposite films by using photo-induced cross-linking. In this study, the starch biocomposite of the films were prepared from the aqueous dispersions of starch with microcrystalline cellulose using glycerol as plasticizer and irradiated under UV light using sodium benzoate as a photosensitizer. The results showed that the tensile modulus and strength were found to improve when photo-irradiation was applied. In summary it is clear that treating starch biocomposite films with photo cross-linking under ultraviolet would improve the physical and mechanical properties of the film.
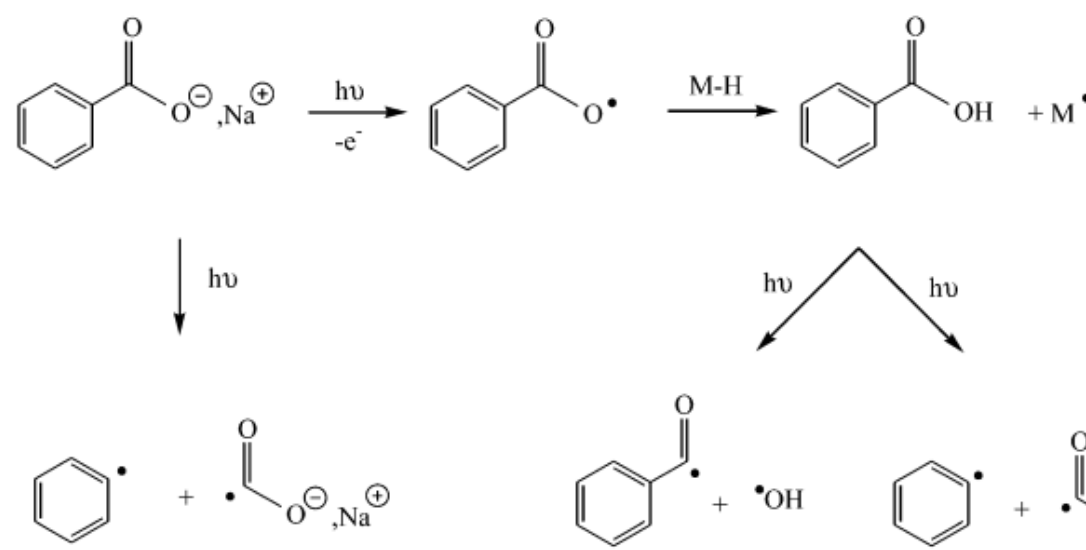<smiles>O=C(O)c1ccccc1</smiles><smiles>O=C(O)[C+]1C=CC=C1</smiles><smiles></smiles><smiles></smiles><smiles></smiles><smiles></smiles><smiles>O=C([IH+])c1ccccc1</smiles>

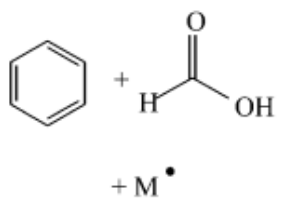

Figure 2. Mechanism involved in sodium benzoate of irradiated rice starch film. Source: Delville et al. (2002) 


\subsubsection{Gamma irradiation treatment}

Gamma irradiation is an ionic, non-heating, processing technology. It has long been used to protect products from insect infestation and microbial contamination during storage and extraction, and to extend the shelf life of foods and medicines (Sabato et al., 2009). Currently, gamma irradiation has become well known as a very convenient tool for the modification of polymer materials through cross-linking, grafting and degradation techniques. This method significantly increases the mechanical properties of films by including cross-links between the starch chains. In addition, gamma irradiation as a cost-lowering and environmentallyfriendly alternative method has been widely applied to produce modified starch.

Some researchers have been investigating the effect of gamma irradiation on the properties of starch films. Kim et al. (2008) studied the effect of gamma irradiation on the physicochemical properties of starch based film. They found that using gamma irradiation in aqueous starch based blends resulted in intact and smooth films. The tensile strength of the film increased at the highest value at $3 \mathrm{kGy}$ and its percent elongation was $85 \%$ higher than its non-irradiated starch film. Furthermore, water vapor permeability of the starch based film was also improved by gamma irradiation. According to these results, gamma irradiation can be a useful tool as a cross-linking agent of starch to improve the functional properties of a starch based film if the optimum irradiation dose is applied. Akter et al. (2012) found that properties of starch and chitosan blend film could improved by using gamma radiation at optimum doses. The improvement of the mechanical and barrier properties of irradiated blend films resulted from the promoting of the cross-linking of polymer during the exposure to radiation. In addition, they concluded that gamma radiation was found to be an excellent method for grafting and cross-linking of synthetic and biodegradable films for packaging.

\subsubsection{Electron beam treatment}

Electron beam irradiation is an excitation process in which radicals are produced by the breaking of the $\mathrm{H}=\mathrm{C}$ bonds of alkene groups. Hence, the monomer molecules, generally multifunctional acrylates, which are in contact with the "activated" material, can copolymerize. The Electron beam irradiation technique is easy to use and control as compared to chemical methods. This technique also displays some advantages over conventional grafting processes including the absence of catalyst residue, complete control over temperature and the absence of a solvent (Peroval et al., 2004). Thus this technique is a green process with potential use in the food field and polymer films. In addition, electron beam irradiation could induce the compatibility of polymers by producing chemical changes in the structure (Olevier et al., 2001). Using this in accord with the starch, it is generally amorphous and mixed with other polymer and/or ingredients to bring about an adequate set of properties for use. The low compatibility between starch polymers in the mixtures is one of the limitations of this approach.

Olivier et al. (2001) reported that the electron beam irradiation of amorphous blends of potato starch and $\mathrm{N}$-allylurea (AU) was a fast process that efficiently impedes the 
spontaneous blooming of mixtures, including large weight fractions of the low molecular weight additive. Pervoral et al. (2004) modified arabinoxylan-based films through grafting of stearyl acrylate by electron beam irradiation. They found that the homogeneous arabinoxylan-based films, which were pre-activated by oxygen plasma and impregnated with a solution of stearyl acrylate before being exposed to an electron beam, had contact angles that increased from $71^{\circ}$ (untreated films) to $122^{\circ}$ (treated films). A decrease of about $24 \%$ in the water vapor permeability was obtained and new chemical groupings were observed on the FTIR spectra of these films. These results were explained by the electron beam inducing the grafting of stearyl acrylate monomers on the arabinoxylan-based films surface and also in the film's internal structure resulting a denser film structure.

\subsubsection{Ultrasonic treatment}

Nowadays, ultrasonic is regarded as an emergent technology in the food, chemical, pharmaceutical and polymer industries. In particular, ultrasonic energy represents a clean way to accelerate and improve the properties of materials. Ultrasonic processing is the use of sound waves beyond the audible frequency range (in general, $>20 \mathrm{kHz}$ ) (Chandrapala et al., 2012). When ultrasonic passes through a liquid medium, the interaction between the ultrasonic waves, liquid and dissolved gas leads to an exciting phenomenon known as acoustic cavitation. Acoustic activation generates chemical reactions; and physical forces that include shear forces, shock waves and turbulence (Ashokkumar et al., 2007). In some applications, the physical forces and chemical reactions are needed. For example, the stability of ultrasonically generated microcapsules that encapsulate drugs and high value material components can be substantially increased by chemically cross-linking starch molecules during the formation process (Cavalieri et al., 2008). One of the major issues in using ultrasound in polymer processing is the controlled modification of the interaction between polymers without chemical modification.

Ultrasonic had been extensively performed in the treatment of starch as early as 1933 (Kardos \& Luche, 2001). From that point on, many researchers have shown that ultrasonic treatment has a great effect on the behaviors of gelatinized starch dispersions. After treating by ultrasound, the starch dispersion showed a decrease in viscosity and an increase in solubility and clarity due to the increase in number of free mobile macromolecules, rather than the breakage of starch molecules (Iida, 2008; Jenny, 2009). These studies suggest that ultrasonic treatment facilitates the disintegration of starch granules and the formation of homogenous starch solution. Compared to conventional dissolution method for starch, ultrasonic treatment is relatively inexpensive and efficient (Liu et al., 2007). The application of ultrasonic treatment to starch film solution could improve the properties of starch films. In addition, ultrasonic provides dispersion capacity and increases the number of free mobile macromolecules resulting in promotion of chain to chain interaction. Cheng et al. (2010) studied the impact of ultrasonic treatment on the properties of maize starch films. They found that the property of maize starch films was affected remarkably by ultrasound treatment procedure previous to casting. Ultrasonic treatment produces an excellent film 
with good transparency, improved moisture resistance and provides stronger structure. The results also showed the resulting films with ultrasound treatment lead to a decrease in strain at break. The improvement of film structure by ultrasonic treatment is probably due to the thorough rupture of starch ghosts and rearrangement of free mobile chains of polymer matrix (mainly amylose and amylopectin) during the drying process is favor of forming a more compact and homogeneous film structure. In addition, the destruction of the starch ghosts and releasing of amylose after ultrasound treatment increases amylose content in casting starch solution. It is known that amylose networks form denser films than amylopectin (Rindlav-Westling et al., 1998) which may be the main reason behind the denser films obtained from ultrasonic treatment.

\subsection{Combination with other materials}

Rice starch can be used to produce biodegradable films to partially or entirely replace plastic polymers because of its low cost and renewability, as well as possessing good mechanical properties ( $\mathrm{Xu}$ et al., 2005). However, wide application of starch film is limited by its mechanical properties and efficient barrier against low polarity compounds. This constraint has led to the development of improved properties of rice films by modifying its starch properties and/or blending it with other materials. Polymer blending is one of the effective techniques for providing new desirable polymeric materials for a variety of applications. The advantages of blending with other materials versus developing new polymer structure are that it can offer the advantage of reducing research and development expense compared to the development of new monomers and polymers to provide a similar profile (Robeson, 2008). Other advantages of polymer blends are that they can offer properties such as profile combinations not easily obtained with new polymer structures. The properties of polymer blends are varied and depend on the type of polymer materials, ratio between polymers, miscibility, and phase behavior. Various polymer materials can be blended with starch based film in order to improve their property. These are described in the following sections.

\subsubsection{Combination with chitosan}

Chitosan is a 1, 4 linked-2-deoxy-2-aminoglucose, prepared by N-deacetylation of chitin $(\mathrm{Xu}$ et al., 2005). Chitosan provides unique functional, nutritional, and biomedical properties, and its present and potential uses range from dietary fiber to a functional ingredient and processing aid. Some of the well known applications of chitosan include its use for prevention of water pollution, treatment against hypertension, antimicrobial and hypocholesterolemic activity, flavor encapsulation, seed coating, film-forming, and controlled release of food ingredients and drugs (Muzzarelli et al., 1997). Its relatively low cost, widespread availability from a stable renewable source such as shellfish waste of the sea food industry, along with chitosan's ability to form a good film, are primary reasons to seek new applications of this polymer. Numerous investigations have been reported on the studies of films made from chitosan (Park et al., 2002) and chitosan blends with starch. 
Zhai et al. (2004) investigated the effect of chitosan on corn starch film properties. The tensile strength and the flexibility of starch film were improved largely after incorporation of $20 \%$ chitosan into starch film. In addition, X-ray diffraction and scanning electron microscope analysis of starch and chitosan blend films indicated that there was interaction between starch and chitosan molecules. Xu et al. (2005) prepared and assessed the starch and chitosan blend films. The film's tensile strength, elongation at break and water vapor transmission rate was affected by the chitosan content. According to the results, the mechanical properties of the composite films increased with the addition of chitosan. The increase in tensile strength with the addition of chitosan may occur because inter-molecular hydrogen bonds between starch and chitosan are formed. However, too high a chitosan content provided inferior mechanical properties. This was because intra-molecular hydrogen bonds occurred rather than inter-molecular hydrogen bonds between polymers.

Bangyekan et al. (2005) investigated the properties of chitosan-coated cassava starch films. They found that coating of chitosan solutions led to an improvement of the mechanical and physical properties of cassava starch films. The results of the evaluation of the mechanical properties showed that an increase in chitosan coating concentration resulted in a significant increase in tensile stress at maximum load and tensile modulus, and a decrease in percent elongation at break. According to the results, it can be concluded that the brittle characteristic of chitosan film may be responsible for an increment of tensile strength and tensile modulus of the coated cassava films.

Bourtoom \& Chinnan (2008) studied the effect of chitosan on biodegradable films made from rice starch. The biodegradable rice starch films showed an increase in tensile strength, water vapor permeability, a decreasing elongation at the break, and film solubility after incorporation of chitosan. Addition, the introduction of chitosan increased the crystalline peak structure of starch film; however, too high a concentration of chitosan yielded phase separation between starch and chitosan. The increasing of the mechanical properties of the biodegradable blend rice starch films, with the addition of chitosan, are attributable to the formation of intermolecular hydrogen bonding between $\mathrm{NH}_{3}{ }^{+}$of the chitosan backbone and $\mathrm{OH}^{-}$of the rice starch. The amino groups $\left(\mathrm{NH}_{2}\right)$ of chitosan were protonated to $\mathrm{NH}_{3}{ }^{+}$in the acetic acid solution, whereas the ordered crystalline structures of starch molecules were destroyed with the gelatinization process, resulting in the $\mathrm{OH}^{-}$groups being exposed to readily formed hydrogen bonds with $\mathrm{NH}_{3}{ }^{+}$of the chitosan. This reaction can be confirmed by the results of the amino group band of the chitosan molecule in the FTIR spectrum which shifted from $1541.15 \mathrm{~cm}^{-1}$ in the chitosan film to $1621.96 \mathrm{~cm}^{-1}$ in the biodegradable blend films. This phenomenon pointed out that interactions were present between the hydroxyl group of rice starch and the amino group of chitosan.An addition of chitosan to rice starch films resulted in increases in the water vapor permeability of the films. This tendency could be explained by the higher hydrophilicity $\left(\mathrm{NH}_{3}{ }^{+}\right.$groups) of the biodegradable blend films

\subsubsection{Combination with polyvinyl alcohol}

Polyvinyl alcohol (PVA) provides excellent film forming, emulsifying, and adhesive properties, which has resulted in its broad industrial use, such as for paper adhesives, a 
textile sizing agent, paper coatings and water soluble films (Ibrahim et al., 2010). PVA is a water soluble polymer; hence PVA can be formed by solution casting and orientation to make high performance PVA films. In addition, PVA can also be resistant to oil, grease and solvents. It is odorless and nontoxic. It has high tensile strength and flexibility, as well as high oxygen and aroma barrier properties. PVA materials show a broad and versatile range of physical properties and other advantageous characteristics at acceptable cost and biodegradation rate and can be employed in a wide range of applications.

The addition of PVA into the starch films to improve their properties (such as mechanical properties, moisture sensitivity) has been reported. Shorgen et al. (1998) reported that the factors that most limit the use of starch in biopolymer material applications are brittleness and loss of strength at low humidity and poor resistance to water. By adding PVA, starch could improve strength and flexibility. However, the desired strength, flexibility, or stiffness can be tailored by formulating with different PVA levels, PVA types, better level of solids and cross-linking agents. Liu et al. (1999) found that incorporation of PVA into starch could enhance the mechanical properties. However, improvement in the mechanical properties is limited, mainly due to the poor interface adhesion between the fibrous PVA structure and the starch matrix. Chen et al. (2008) investigated the effect of PVA on the properties of starch films. It was found that the tensile strength, elongation at break of starch films increased with the addition of PVA. Because both starch and PVA are polar substances having hydroxyl groups $(-\mathrm{OH})$ in their chemical structure, these highly polar hydroxyl groups tend to form inter molecular and intra-molecular hydrogen bonds which improve the integrity of starch-PVA films. On the other hand, the solubility parameters of starch and PVA modeling complexes have been found to be close to each other, which confirmed that starch and PVA are compatible blends. In addition, the vibration frequency analysis of these molecular complexes has shown that the hydroxyl group shifted to lower wave numbers due to formation of hydrogen bonds between starch and PVA.

\subsubsection{Combination with polylactic acid}

Polylactic acid (PLA) is a rigid degradable thermoplastic polymer which can be semicrystalline or totally amorphous, depending on the stereochemistry of the polymer backbone. PLA is a unique polymer that has, in many ways, properties like PET, but also performs much like polyolefin. PLA have been used in biomedical fields, for example, as a surgical implant material and drug delivery system, as well as in textile applications. This is because of their excellent properties such as biocompatibility, nontoxicity, high mechanical strength, and thermal plasticity (Lertworasirikul et al., 2008). In addition, PLA also exhibits excellent characteristics and is suitable for food contact surface and related packaging applications.

PLA can be formed into transparent films and fibers. Certain factors suggest the incorporation of PLA into rice starch films would bring benefits and improve their properties. These include relatively reasonable costs and specifically, biocompatibility, nontoxicity, high mechanical strength, and thermal plasticity. Research has been reported, such as Ke \& Son (2003) that suggests that PLA performed as a reinforcement in the starch. 
These results showed that the tensile strength and elongation of the starch blend films increased when PLA was added. Yew et al. (2005) studied the effect of PLA on the properties of rice starch films and found that the tensile strength of the rice starch increased with the incorporation of PLA. This may be due to the polar interaction between the starch and carboxyl groups of the PLA. In addition, the interaction between PLA and rice starch may be attributed to the possible hydrogen bonding that occurs between the carbonyl group (that is from ester linkage) in PLA and the hydroxyl group in starch. A proposed possible site for interaction between starch and PLA is depicted in Figure 3.

Polylactic acid (PLA)<smiles>CCOC(=O)C(C)OC(=O)C(C)OC(=O)C(C)O</smiles>

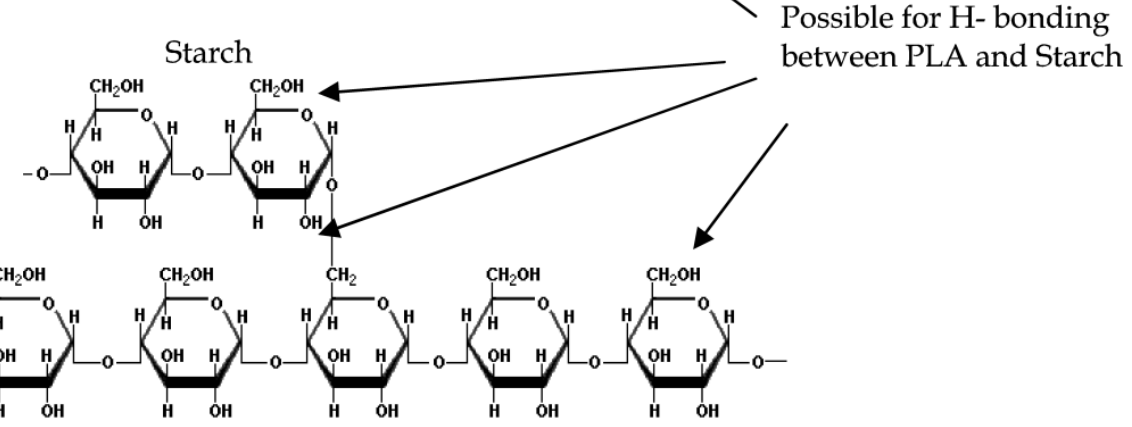

Figure 3. Proposed chemical interactions between starch and PLA. Source: Modified from Yew et al. (2005)

\subsubsection{Combination with algae polysaccharides}

Algae are photosynthetic microorganisms containing chlorophyll. They can be single cell or multicell, motile or non-motile. Algae and their extracts are currently used in medicine, food, and the nutraceutical, pharmaceutical and packaging industries. Most of the world's annual seaweed harvest is used to produce the algal hydrocolloids and those that show potential for application in biodegradable films are alginate, agar, and carrageenan. Alginates are natural substances extracted from brown seaweed. In polymer chains, monomers are arranged alternately in GG and MM blocks, together with MG blocks (Grant, 1973). The most interesting property of alginates is their ability to react with polyvalent metal cations, specifically calcium ions. The alginate films show hydrophilic matrices, and the cross-linking process with polyvalent cations has been used to improve their water barrier properties, mechanical resistance, cohesiveness and rigidity (Rhim, 2004). Due to the fast cross-linking process between alginate and calcium ions, localized gelling areas are produced, compromising the uniformity and quality of films. While, agar is composed of 
alternating 1, 3-linked-D-galactose and 1,4- linked 3,6- anhydro-L-galactose units. It is substituted by sulfate esters and methoxyl, and may also carry pyruvic acid residues. Because of its ability to form very hard gels at very low concentrations, agar has been used extensively as a gelling agent in the food industry. Because of its combination of renewability and biodegradability, its enormous gelling power, and the simplicity of the extraction process, agar has been singled out as a promising candidate for future use in plastic materials. Currently, agar has been incorporated in materials such as foams, films and coatings (Phan et al., 2009), and added into starch films to improve the puncture strength and water barrier (Letendre et al., 2002).

Carrageenan is biopolymers extracted from algae, are used extensively in the food and packaging industry. The three main carrageenans differ only in the number of sulphate groups. k-Carrageenan is the most sulphated one and adopts a coil conformation whatever the ionic and temperature conditions. However, kappa and iota carrageenan form gels, but lambda is unable to do this and is used as a pure thickener (Lizarraga et al., 2006). kCarrageenan itself is known for its good film forming ability (Choi et al., 2005).

The algae polysaccharide could provide good film and shows the potential to improve the properties of starch. Hence, the addition of algae polysaccharide into starch films for improvement of properties has been suggested. Wu et al. (2009) assessed the effect of agar on potato starch films; it was found that the addition of agar could enhance the tensile strength and water barrier of the resulting films. The increase in their properties was attributable to the formation of inter-molecular hydrogen bonds between starch and agar and the compact structure of composite film. In addition, the domains occurred of the threedimensional network structure formed by entanglement among agar chains. The water barrier properties of the starch film were promoted when agar was added; this was due to the strong inter-molecular interaction formed between starch and agar. It minimized the free volume and inter-molecular distances in the film structures. Consequently, water molecules were diffused with greater difficulty in the compact network and a higher water barrier value was obtained.

In addition, Phan et al. (2009) reported that adding agar into cassava starch film provided an improvement in elongation at break and the tensile strength of cassava starch film. This indicates that incorporation of agar into starch film could provide a very good cohesive matrix, which contributes to enhancing the mechanical properties of starch based films. Cordoba et al. (2008) studied the effect of alginate on the properties of thermoplastic starch. According to the experimental evidence presented in their work, a decrease in the elastic properties and an increase in elongation at break and impact resistance was observed when alginate was added into the thermoplastic starch. These results could be explained by the addition of alginate to thermoplastic starch causing an obvious plasticizing effect. However, the effect of the amount of alginate content on thermoplastic starch when used for specific needs and applications should be determined. Lafargue et al. (2007) determined the influence of k-Carrageenan on film forming and film properties of modified starch. This study demonstrated that the addition of a low amount of the carrageenan into starch 
resulted in an enhancement of film solution properties and thermo-reversibility without modifying the film properties. According to the results, it might be beneficial to design tailor-made films from starch by using carrageenan.

\subsection{Reinforcement}

\subsubsection{Reinforcement with natural fibers}

Narural fibers can be considered as naturally occurring composites, consisting of cellulose fibrils embedded in lignin matrix. The cellulose fibrils are aligned along the length of the fiber, which render maximum tensile and flexural strengths, in addition to providing rigidity. Natural fibers are, in general, suitable to reinforce materials due to their relatively high strength and stiffness and low density. Natural fibers can be processed in different ways to yield reinforcing elements with different mechanical properties. Natural fiberreinforced starch film has many advantages such as light weight, reasonable strength, stiffness and thermal resistance (Demir et al., 2006; Phataraporn et al., 2010). This behavior has been attributed to the resistance exerted by the fiber itself and also due to 3D hydrogen bonds network formed by intermolecular interactions between starch and fiber, which reduces the flexibility of molecular chains of starch ( $\mathrm{Lu}$ et al., 2006). Furthermore, there is compatibility between the starch matrix and fiber and performance (such as the mechanical properties). This behavior was confirmed by observing different polysaccharides reinforced with cellulose fiber. Various types of fiber have been applied in starch based polymer films, such as bleached leaf wood fibers, bleached eucalyptus pulp fibers, wood pulp softwood aspen, jute, hemp and flax fibers, tunicin whiskers, and oil palm fibers. It is known that fiber related parameters such as their dispersion, length, type, size, content of natural fiber and their orientation along with their adhesion with the matrix all determine the properties of their composites (Goda et al., 2006).

\subsubsection{Reinforcement with modified natural fibers}

Reinforcing fibers can be modified by physical and chemical methods. Physical methods, such as stretching, calendaring, thermo-treatment and the production of hybrid yarns do not change the chemical composition of the fibers. Physical treatment changes structure and surface properties of the fiber and thereby influence the mechanical bondings to polymers. With regards to the chemical methods, the strongly polarized cellulose fibers are inherently incompatible with hydrophobic polymers. When two materials are incompatible, it is often possible to bring about compatibility by introducing a third material that has properties intermediate between those of the other two. There are several mechanisms for coupling in materials: (1) weak boundary layers - coupling agents eliminate weak boundary layers; (2) deformable layers - coupling agents produce a tough, flexible layer; (3) restrained layers coupling agents develop a highly cross-linked inter-phase region, with a modulus intermediate between that of substrate and of the polymer; (4) chemical bonding - coupling agents form covalent bonds with both materials; and (5) acid - base effect - coupling agents alter the acidity of the substrate surface. In relation to the coupling agent used in fiber 
modification, organosilanes are the main groups of coupling agents. They have been developed to couple virtually any polymer to the minerals used in reinforced composites. Most of the silane coupling agents can be represented by the following formula:

$$
\mathrm{R}-\left(\mathrm{CH}_{2}\right)_{\mathrm{n}}-\mathrm{Si}\left(\mathrm{OR}^{\prime}\right)_{3}
$$

where $n=0-3, O R^{\prime}$ is the coupling agent that causes the reaction with the polymer. This could be co-polymerization, and/or the formation of an interpenetrating network.

The curing reaction of a silane treated substrate enhances the wetting by the resin. HerreraFranco \& Valadez-Gonzalez (2005) investigated the properties of short natural fiber reinforced composites as a function of chemical modification. Short natural fibers were subjected to silane treatment (vinyltris or 2-methoxy-ethoxy silane). The authors observed that the increase in the mechanical properties ranged between 3 and $30 \%$, for the tensile and flexural properties. The shear strength of this composite showed an increase of the order of $25 \%$. From the micrographs, obtained from failure surfaces from the SEM, it was observed that with increasing fiber-matrix interaction the failure mode changed from interfacial failure to matrix failure. The interface failure was mainly a frictional type failure, and matrix tearing and shearing was observed only for the pre-impregnated and silane treated fibers. The silane surface treated fibers also showed a layer of polymer covering the fibers even after failure.

Shih (2007) has also developed epoxy composites from waste water bamboo husk fiber. The fibers were chemically modified by coupling agents and untreated fibers were added to epoxy resin to form new reinforced composites. The results show that the morphology analysis reveals that the fibers modified by coupling agent exhibited better compatibility with the polymer matrices than the untreated fiber. In addition, the mechanical properties were also enhanced due to the addition of fibers treated with coupling agents and untreated fibers. The increments of storage modulus of epoxy were about 16.4 and $36.1 \%$ with the addition of $10 \%$ in the fibers treated with coupling agents and untreated fibers, respectively.

Phattaraporn et al. (2011) studied the effect of palm pressed fiber (PPF) surface treatment on the properties of rice starch films. They reported that higher tensile strength, water vapor permeability and thermal properties of biodegradable rice starch films were obtained as silane treated PPF was applied. Increasing the concentration of silane and content of silane treated (PPF) resulted in increased tensile strength and water vapor permeability but decreased elongation at break. In addition, the glass temperature $\left(\mathrm{T}_{\mathrm{g}}\right)$ shifted towards higher temperatures with an increasing concentration of silane. This could be a restriction of the mobility of the starch chain due to the establishment of strong interactions between rice starch films and treated PPF. The maximum improvement of rice starch films in the mechanical and thermal properties was obtained when $40 \%$ of silane treated PPF films was applied. These results pointed out that the interfacial interactions improved the filler compatibility, and the mechanical and thermal properties. In addition, the treatment of fiber with alkaline $(\mathrm{NaOH})$ leads to a decrease in the spiral angle, that is it is closer to the fiber axis, and and increase in molecular orientation. 


\subsubsection{Reinforcement with nanoparticles}

\subsubsection{Nanoclays}

Nanoclays are potential filler. It is a naturally abundant mineral that is toxin-free and can be used as one of the components for food, medical use, cosmetics, healthcare products and packaging. Moreover, nanoclays are also environmentally friendly and inexpensive. Nanoclays have also been reported to improve the mechanical strength of biopolymers, making their use feasible. The structure and properties of the resulting material is dependent on the state of the nanoclay in the nanocomposite, that is if it is exfoliate or intercalate. Intercalation is the state in which polymer chains are present between the clay layers, resulting in amultilayered structure with alternating polymer/inorganic layers. Exfoliation is a state in which the silicate layers are completely separated and dispersed in a continuous polymer matrix (Weiss et al., 2006). The most common nanoclay used in composites with starch films is montmorillonite (MMT). Because of the presence of sodium cations between the interlayer spaces, natural MMT is hydrophilic and is miscible with hydrophilic polymers, such as starch. Addition, starch-MMT nanocomposites have gained prominence due to the mechanical reinforcement achieved, even at low concentrations (Ardakani et al., 2010).

Many report that nanoclays show improvement in the mechanical strength of starch films, making their use a possibility. Chung et al. (2010) improved the properties of starch film by adding nanoclay (montmorillonite) into the film solution. The addition of nanoclay in starch film show improved modulus and strength without a decrease in elongation at break. The increase in modulus and strength is $65 \%$ and $30 \%$, respectively for the starch film containing $5 \mathrm{wt} . \%$ nanoclay compared to the unfilled starch materials. However, further increases in clay result in deterioration in properties that are most likely due to poorer clay dispersion and lower polymer crystallinity.

Muller et al. (2011) studied the effect of nanoclay on mechanical and water vapor barrier properties of starch films. The nanocomposites exhibited a remarkable improvement in mechanical properties and reduced the water vapor permeability when compared with pure starch films. This behavior may be associated with the interaction between nanoclay and the molecules of starch. Gao et al. (2012) studied the effect of nanoclays on the properties of starch film. It was found that the addition of clays, significantly greater tensile strength and lower water vapor permeability were obtained. Besides, starch-clay composite films exhibited a lower glass transition temperature $\left(\mathrm{T}_{\mathrm{g}},-23.8{ }^{\circ} \mathrm{C}\right)$ and better heat endurance than unfilled starch film. They suggested that the coupling between the tremendous surface area of the clay and the starch matrix facilitated the stress transfer to the reinforcement phase, allowing for the tensile and toughening improvements.

\subsubsection{Nanocrystalline cellulose}

Crystalline cellulose is obtained by hydrolysis of the amorphous portion of cellulose until a level-off in the degree of the product of polymerization is obtained. Crystalline cellulose is comprised of highly crystalline regions of cellulose. It is useful for a number of different 
applications. Pieces of crystalline cellulose easily bond together without the use of an adhesive. Furthermore, crystalline cellulose can be mixed with other substances so as to hold an additive while bonding to itself. It can be made into pharmaceutical-grade tablets, such as those used for vitamins, pain relievers and other medicines. It also may be used as a substitute for starch where starch is used as a smoothener, such as in suntan lotion.

Due to its high aspect ratio and a high modulus, the use of cellulose crystallites for preparation of high performance composite materials has been explored extensively. When the cellulose crystallites were homogeneously dispersed into polymer matrices, they gave a remarkable reinforcing effect, even at concentrations of a few percent (Favier et al., 1996). The hydrolysis of cellulose to obtain crystalline cellulose can be accomplished using mineral acid, enzymes or microorganisms. Although such methods are desirable because glucose, a useful by-product is created, these methods are more expensive and create crystalline cellulose products with a lower crystallinity. Thus, acid hydrolysis is the conventional method of choice for manufacturing crystalline cellulose. Acid that can be used in this process includes hydrochloric acid, sulfuric acid and phosphoric acid. At higher temperatures, sulfuric acid and phosphoric acid can peptize and modified surface crystalline cellulose. Nanocrystalline cellulose is inherently a low cost ingredient and provides a strength of about $10 \mathrm{GPa}$ values which are only about seven times lower than those of single-walled carbon nanotubes (Podsiadlo et al., 2005). Thus, nanocrystalline cellulose shows a high potential application for starch film improvement.

$\mathrm{Lu}$ et al. (2006) investigated the morphological and mechanical properties of ramie crystallites (RN) reinforced plasticized starch (PS) biocomposites. The ramie cellulose nanocrystalites, having lengths of $538.5 \pm 125.3 \mathrm{~nm}$ and diameters of $85.4 \pm 25.3 \mathrm{~nm}$ on average, were prepared from ramie fibers by acid hydrolysis. The results indicate that the synergistic interactions between fillers and between filler and matrix play a key role in reinforcing the composites. The PS/RN composites, composites conditioned at $50 \%$ relative humidity, increases in tensile strength and Young's modulus from 2.8 MPa for PS film to 6.9 $\mathrm{MPa}$ and from $56 \mathrm{MPa}$ for PS film to $480 \mathrm{MPa}$ with increasing RN content from 0 to $40 \mathrm{wt} \%$ respectively. Furthermore, incorporating RN fillers into PS matrix also leads to a decrease in water sensitivity for the PS based biocomposites. Alemdar \& Sain (2007) later studied the morphology, thermal and mechanical properties of wheat straw nanofibers as reinforced starch biocomposites. The nanocomposites from the wheat straw nanofibers and the thermoplastic starch were prepared by the solution casting method. They founded that the tensile strength and modulus of the nanocomposite films showed significantly enhanced properties compared to the pure thermoplastic starch. The glass transition of the nanocomposites was shifted to higher temperatures with respect to the pure thermoplastic starch.

Wittaya et al. (2009) reinforced the rice starch films with microcrystalline cellulose (average length of about $0.480 \pm 0.023 \mu \mathrm{m}$ ) from palm pressed fiber. The strong interactions between microcrystalline cellulose from palm pressed fiber and between microcrystalline cellulose from palm pressed fiber and rice starch film matrix played a key role in reinforcing the 
resulting rice starch film composites. The rice starch film/ microcrystalline cellulose from palm pressed fiber biocomposites increased in tensile strength from $5.16 \mathrm{MPa}$ for pure rice starch film to $44.23 \mathrm{MPa}$ but decreased in elongation at the breaking point of the composites. In addition, the incorporation of microcrystalline cellulose from palm pressed fiber into rice starch films provided an improvement of the water resistance for the rice starch films.

\subsubsection{Starch nanocrystals}

Starch nanocrystal is prepared by submitting native starch granules to an extended time of hydrolysis at a temperature below the gelatinization temperature. This is when the amorphous regions are hydrolyzed allowing the separation of crystalline lamellae, which are more resistant to hydrolysis. The starch crystalline particles show platelet morphology with thicknesses of 6-8 $\mathrm{nm}$ (Kristo \& Billiaderis, 2007). The use of starch nanoparticles is receiving a significant amount of attention because of the abundant availability of starch, low cost, renewability, biocompatibility, biodegradability and non-toxicity. The latter properties make them excellent candidates for implant materials and drug carriers. Kristo \& Biliaderis (2007) studied the addition of starch nanocrystals on the properties of pullulan film. The water uptake of pullulan-starch nanocomposites decreased with increasing filler content whereas water vapor permeability remained constant up to $20 \%(\mathrm{w} / \mathrm{w})$. This then decreased significantly with further addition of starch nanocrystals. The thermo-mechanical behavior of nanocomposite films was also investigated by means of dynamic mechanical thermal analysis (DMTA) and large deformation mechanical tests. The glass transition temperature $\left(\mathrm{T}_{\mathrm{g}}\right)$ shifted towards higher temperatures with increasing amounts of nanocrystals. This can be attributed to a restriction of the mobility of pullulan chains due to the establishment of strong interactions not only between starch nanocrystals but also between the filler and the matrix. Moreover, the addition of nanocrystals caused strong enhancement of the Young modulus and the tensile strength, but led to a drastic decrease of the strain at break in the samples

Chen et al. (2007) prepared pea starch nanocrystal (PSN) dispersion containing nanocrystals within a range of 30-80 nm from native pea starch (NPS) granules by acid hydrolysis. The addition of 5 and $10 \mathrm{wt} \%$ of PSN content gave improved physical properties compared to the PVA film. The PVA/PSN films showed higher tensile strength, elongation at break and lower moisture uptake than the corresponding PVA/NPS films with the same components. The results revealed that PSN, comparing with NPS, had much smaller sizes and dispersed more homogeneously in the PVA matrix, resulting in stronger interactions with PVA. New applications of native pea starch and its nanocrystals as low-cost fillers were explored in this work, and PSN showed greater potential than NPS to improve the properties of PVA-based composites.

Kaewpool et al. (2010) improved the rice starch films properties by addition of starch nanocrystals. The results showed that the mechanical properties of the rice starch film were enhanced by the addition of starch nanocrystals. This indicated that introduction of starch nanocrystals increased the crystalline peak structure of rice starch film. Furthermore, by 
increasing the starch nanocrystals content, the water barrier properties of the rice starch films were also improved. The glass transition temperature $\left(\mathrm{T}_{\mathrm{g}}\right)$ shifted towards higher temperatures when increasing the amount of nanocrystals and the char yield of rice starch films was enhanced as the starch nanocrystals increased. Therefore, the addition of starch nanocrystals to rice starch films can improve the thermal stability of rice starch films. The results demonstrated that the addition of starch nanocrystals by $15 \%$ provided better properties, including mechanical, water barrier and thermal properties.

\section{Conclusion}

Biodegradable film made from rice starch shows potential for use as packaging material. However, compared to the common thermoplastics, biodegradable rice starch films still reveal some disadvantages, such as low mechanical properties and lack of efficient barriers against polarity compounds. This constraint has led to the development of the improved properties of biodegradable film from rice starch by modifying its starch properties and/or incorporating other materials. Chemical treatments and physical treatment, in combination with other biodegradable materials and fiber reinforcement could improve the properties of biodegradable film made from rice starch. However, the potential application, in terms of properties and limitations on the use of facilities, should be taken into account when selecting techniques for improvement.

\section{Author details}

Thawien Wittaya

Prince of Songkla University, Department of Material Product Technology, Thailand

\section{References}

Akter, N.; Lacroix, M.; Salmieri, S.; Dussault, D.; Khan, R. \& Sharmin, N. (2012). Effectiveness of silane monomer and gamma radiation on chitosan films and PCL-based composites. Physics and Chemistry, In press. (January 2012), ISSN: 0969-806X.

Alemdar, A. \& Sain, M. (2008). Biocomposites form wheat straw nanofiber: Morphology, thermal and mechanical properties. Composite Science and Technology, Vol.68, No. 2, (February 2008), pp. 557-565, ISSN: 0266-3538.

Alves, D. V.; Mali, S.; Beleia, A. \& Grossmann, E. M. (2007). Effect of glycerol and amylose enrichment on cassava starch film properties. Journal of Food Engineering, Vol. 78, No. 3 (February 2007), pp. 941-946. ISSN: 0260-8774.

Ardakani, M. M.; Mohseni, M. A.; Beitollahi, H.; Benvidi, A. \& Naeimi, H. (2010). Electrochemical determination of vitamin $\mathrm{C}$ in the presence of uric acid by a novel $\mathrm{TiO} 2$ nanoparticlesmodified carbon paste electrode. Chinese Chemical Letters, Vol.21, No.12, (December 2010), pp. 1471-1474. ISSN: 1001-8417. 
Ashokkumar, M.; Sunartio, D.; Kentish, S.; Mawson, R.; Simons, L.; Vilkhu, K. \&Versteeg, C. (2008). Modification of food ingredients by ultrasound to improve functionality: a preliminary study on a model system. Innovative Food Science Emerging Technology, Vol.9, No.2, (April 2008), pp. 155-160. ISSN: 1466-8564.

Bangyekan, C.; Aht-Ong, D. \& Srikulkit, K. (2006). Preparation and properties evaluation of chitosan-coated cassava starch films. Carbohydrate Polymers, Vol.63, No.1, (January 2006), pp. 61-71. ISSN: 0144-8617.

Banker, G. S. (1966). Film coating theory and practice. Pharmaceutical Sciences, Vol.55, No.1, (January 1966), pp. 81-89. ISSN: 1520-6017.

Bhat, R. \& Karim, A. A. (2009). Impact of radiation processing on starch. Comprehensive Reviews in Food Science and Food Safety, Vol. 8, No. 2 (March 2009), pp. 44-58. ISSN: 15414337.

Bintsis, T.; Litopoulou-Tzanetaki, E. \& Robinson, R. K. (2000). Existing and potential applications of ultraviolet light in the food industry- A critical review. Science of Food and Agriculture, Vol.80, No.6, (May 2000), pp. 637-645. ISSN: 1097-0010.

Bourtoom, T. \& Chinnan, M. S. (2008). Preparation and properties of rice starch-chitosan blend biodegradable film. Lebensmittel-Wissenschaft Und-Technologie, Vol.41, No.9, (November 2008), pp. 1633-1641. ISSN: 0023-6438.

Bourtoom, T. \& Chinnan, M. S. (2009). Improvement of water barrier property of rice starchchitosan composite film incorporated with lipids. Food Science and Technology International, Vol. 15, No. 2, (June 2009), pp. 149-158. ISSN: 1082-0132.

Cavalieri, F.; Ashokkumar, M.; Grieser, F. \& Caruso, F. (2008). Ultrasonic synthesis of stable functional lysozyme microbubbles. Langmuir, Vol.24, No.18, (September 2008), pp. 10078-10083. ISSN: 1520-5827.

Chandrapala, J.; Zisu, B.; Palmer, M.; Kentish, S. \& Ashokkumar, M. (2011). Effects of ultrasound on the thermal and structural characteristics of proteins in reconstituted whey protein concentrate. Ultrasonics Sonochemistry, Vol.18, No.5, (September 2011), pp. 951-957. ISSN: 1350-4177.

Chen, Y.; Stipanovic, A. J.; Winter, W. T.; Wilson, D. B. \& Kim, Y. J. (2007). Effect of digestion by pure cellulases on crystallinity and average chain length for bacterial and microcrystalline celluloses. Cellulose, Vol.14, No.4, (August 2007), pp. 283-293. ISSN: 1572-882X.

Chen, L. S. \& Lai, C. H., (2008). Mechanical and water vapour barrier properties of tapioca starch/decolorized hsian-tsao leaf gum films in the presence of plasticizer. Food Hydrocolloids, Vol.22, No.8, (December 2008), pp. 1584-1595. ISSN: 0268-005X.

Cheng, W. J.; Chen, J. C.; Liu, D. H.; Ye, X. Q. \& Ke, F. S. (2010). Impact of ultrasonic treatment on properties of starch film-forming dispersion and the resulting films. Carbohydrate Polymers, Vol.81, No.3, (July 2010), pp. 707-711. ISSN: 0144-8617.

Choi, J. H.; Choi, W. Y.; Cha, D. S.; Chinnan, M. S.; Park, H. J. \& Lee, D. S. (2005). Diffusivity of potassium sorbate in kappa-carrageenan based antimicrobial film. LebensmittelWissenschaft Und-Technologie, Vol.38, No.4, (June 2005), pp. 417-423. ISSN: 0023-6438. 
Chung, Y.; Ansari, S.; Estevez, L.; Hayrapetyan, S.; Giannelis, E. P. \& Lai, H. M. (2010). Preparation and properties of biodegradable starch-clay nanocomposites. Carbohydrate Polymers, Vol.79, No.2, (January 2010), pp. 391-396. ISSN: 0144-8617.

Clopeau, M. \& Prunet-Foch, B. (1990). Electrostatic spraying of liquids. Main functioning modes. Electrostatics, Vol.25, No.2, (October 1990), pp. 165-184. ISSN: 0304-3886.

Cordoba, A.; Cuellar, N.; González, M. \& Medina, J. (2008). The plasticizing effect of alginate on the thermoplastic starch/glycerin blends. Carbohydrate Polymers, Vol.73, No.3, (August 2008), pp. 409-416. ISSN: 0144-8617.

Dai, H.; Chang, P.R.; Geng, F.; Yu, J. \& Ma, X. (2010). Preparation and properties of starchbased film using N,N-bis(2-hydroxyethyl) formamide as a new plasticizer. Carbohydrate Polymers, Vol.79, No.2, (January 2010), pp. 306-311. ISSN: 0144-8617.

Das, K.; Ray, D.; Bandyopahyay, N. R.; Gupta, A.; Sengupta, S.; Sahoo, S.; Mohanty, A., \& Misra M. (2010). .Preparation and Characterization of Cross-Linked Starch/Poly(vinylalcohol) Green Films with Low Moisture Absorption. Industrial and Engineering Chemistry Research, Vol.49, No.5, (March 2010), pp. 2176-2185. ISSN :15205045.

Delville, J.; Joly, C.; Dole, P. \& Bliard, C. (2002). Solid state photo-crosslinked starch based films: a new family of homogeneous modified starches. Carbohydrate Polymers, Vol.49, No.1, (July 2002), pp. 71-81. ISSN: 0144-8617.

Demir, H.; Atikler, U.; Balkose, D. \& Tihminlioglu, F. (2006). The effect of fiber surface treatmentson the tensile and water sorption properties of polypropylene luffa fiber composites. Composite Part. A, Vol.37, No.3, (March 2006), pp. 447-456. ISSN: 1359-835X.

El-Tahlawy, K.; El-Nagar, K. \& Elhendawy, A. G. (2007). Cyclodextrin-4 hydroxy benzophenone inclusion complex for UV protective cotton fabric. Textile Institute, Vol.98, No.5, (2007), pp. 453-462. ISSN: 1754-2340.

Favier, V.; Chanzy, H. \& Cavaille, J. Y. (1995). Polymer nanocomposites reinforced by cellulose whiskers. Macromolecules, Vol.28, No.18, (August 1995), pp. 6365-6367. ISSN: 1520-5835.

Follain, N.; Joly, C.; Dole, P.; \& Bliard, C. (2005). Properties of starch based blends. Part 2. Influence of poly vinyl alcohol addition and photo-crosslinking on starch based materials mechanical properties. Carbohydrate Polymers, Vol.60, No.2, (May 2005), pp.185-192. ISSN: 0144-8617.

Gao, W.; Dong, H.; Hou, H. \& Zhang, H. (2012). Effects of clays with various hydrophilicities on properties of starch-clay nanocomposites by film blowing. Carbohydrate Polymers, Vol.88, No.1, (March 2012), pp. 321-328. ISSN: 0144-8617.

Gehring, J. (2000). With radiation cross linking of engineering plastics into the next millennium. Radiation Physics and Chemistry, Vol. 57, No.3-6 (March 2000), pp. 361-365. ISSN: 0969-806X.

Ghosp, P. \& Gangopadhay, R. (2000). Photo functionalisation of cellulose and lignocelluloses fibres using photoactive organic acids. European Polymer, Vol.36, No.3, (March 2000), pp. 625-634. ISSN: 0014-3057.

Goda, K.; Sreekala, M.S.; Gomes, A.; Kaji, T. \& Ohgi, J. (2006). Improvement of Plant Based Natural Fibers for Toughening Green Composites-effect of Load Application During 
Mercerizationof Ramie Fibers. Composites: Part A, Vol.37, No.12, (December 2006), pp. 2213-2220. ISSN: 1359-835X.

Grant, G. T.; Morris, E. R.; Rees, D. A.; Smith, P. J. C. \& Thom, D. (1973). Biological interactions between polysaccharides and divalent cations: the egg-box model. FEBS letters, Vol 32, No. 1 (May 1973), pp. 195-198. ISSN: 0014-5793.

Greener, J. K. \& Fennema, O. (1989). Barrier properties and surface characteristics of edible bilayer films. Food Science, Vol.54, No.6, (November 1989), pp. 1393-1399. ISSN: 17503841.

Guilbert, S. (1986). Technology and application of edible protective films. In: Food packaging and preservation, Theory and Practice, Matholouthi, M., pp. 371-394. Elsevier Applied Science Publishing, ISSN: 0853343772, London.

Haggenmaier, R. D. \& Shaw, P. E. (1990). Moisture permeability of edible films made with fatty acid and (hydrocypropyl) methylcellulose. Agricultural and Food Chemistry, Vol.38, No.9, (September 1990), pp.1799-1803. ISSN: 0021-8561.

Herrera-Franco, P. J. \& Valadez-Gonzalez, A. (2005). A. study of the mechanical properties of short naturalfiber reinforced composites. Composite. Part B Engineering, Vol. 36, No.8, (December 2005), pp. 597-608. ISSN: 1359-8368.

Hernandez, R. J. (1994). Effect of water vapor on the transport properties of oxygen through polyamide packaging materials. Food Engineering, Vol.22, No.1-4, (1994), pp. 495-507. ISSN: 0260-8774.

Hoseney, R. C. (1986). Principles of Cereal Science and Technology. Cereal Chemistry, Vol. 63, No. 3 ( May 1986), pp. 285-286. ISSN: 0009-0352

Iida, Y.; Tuziuti, T.; Yasui, K.; Towata, A. \& Kozuka, T. (2008). Control of viscosity in starch and polysaccharide solutions with ultrasound after gelatinization. Innovative Food Science and Emerging Technologies, Vol.9, No.2, (April 2008), pp. 140-146. ISSN: 14668564.

Ibrahim, M. M.; El-Zawawy, W. K. \& Nassar, M. A. (2010). Synthesis and characterization of polyvinyl alcohol/nanospherical cellulose particle films. Carbohydrate Polymers, Vol.79, No.3, (February 2010), pp. 694-699 ISSN: 0144-8617.

Jenny, Y. Z.; Kai, K.; Raymond, M.; Sandra, K. \& Muthupandian, A. (2009). The pasting properties of sonicated waxy rice starch suspensions. Ultrasonics Sonochemistry, Vol.16, No.4, (April 2009), pp. 462-468. ISSN: 1350-4177.

Jordan, J.; Jacob, K. I.; Tannenbaum, R.; Sharaf, M. A. \& Jasiuk, I. (2005). Experimental trends in polymer nanocomposites: a review. Materials Science and Engineering A, Vol. 393, No.1-2, (February 2005), pp. 1-11. ISSN: 0921-5093.

Kaewpool, P. \& Witthaya, T. (2010). Properties and Characteristics of Rice Starch Films Reinforced with Rice Starch Nanocrystals, Proceeding of International Conference on Applied Science Engineering and Technology, Conference Venue River View Hotel. Singapore, August 2010.

Kardos, N. \& Luche, J. L. (2001). Sonochemistry of carbohydrate compounds. Carbohydrate Research, Vol.332, No.2, (May 2001), pp. 115-131. ISSN: 0008-6215. 
Kasemsuwan, T. \& Jane, J. (1996). A quantitative method for the analysis of phosphorus structures and contents in starch by P-31 nmr spectroscopy. Cereal Chemistry, Vol. 73, No.6, (1996), pp. 702. ISSN: 0009-0352.

Ke, T. \& Sun, X.S. (2003). Starch, poly(lactic acid), and poly(vinyl alcohol) blends. Polymers and the Environment, Vol.11, No.1, (January 2003), pp.7-14. ISSN: 1572-8900.

Kerr, R. W. \& Cleveland Jr., F. C. (1962). Thickening agent and method of making the same. United States Patent, 3,021,222. ISSN: 0161-9470.

Kester, J. J. \& Fennema, O. R. (1986). Edible films and coatings: Areview. Food Technology, Vol. 40, No.12, (December 1986), pp.47-59. ISSN: 1365-2621.

Khan, M. A.; Bhattacharia, S. K.; Kader, M. A. \& Bahari, K. (2006). Preparation and characterization of ultra violet (UV) radiation cured bio-degradable films of sago starch/PVA blend. Carbohydrate Polymers, Vol.63, No.3, (March 2006), pp. 500-506. ISSN: 0144-8617.

Kim, J. Y.; Han, S. II. \& Hong, S. (2008). Effect of modified carbon nanotube on the properties of aromatic polyester nanocomposites. Polymers, Vol.49, No.15, (July 2008), pp. 33353345. ISSN: 0032-3861.

Kim, M. \& Lee, S. J. (2002). Characteristics of cross linked potato starch and starch-filled linear low-density polyethylene films. Carbohydrate Polymers, Vol.50, No.4, (December 2002), pp. 331-337. ISSN:0144-8617.

Kristo, E. \& Biliaderis, C. G. (2007). Physical properties of starch nanocrystal reinforced pullulanfilms. Carbohydrate Polymers, Vol.68, No.1, (March 2007), pp. 146-158. ISSN: 0144-8617.

Kumar, A. P. \& Singh, R. P. (2008). Biocomposites of cellulose reinforced starch: Improvement of properties by photo-nducedcrosslinking. Bioresource Technology, Vol.99, No.8, (December 2008), pp. 8803-8809. ISSN: 0960-8524.

Lafargue, D.; Lourdin, D. \& Doublier, J. L. (2007). Film-forming properties of a modified starch/k-carrageenan mixture in relation to its rheological behaviour. Carbohydrate Polymers, Vol.70, No.1, (August 2007), pp. 101-111. ISSN: 0144-8617.

Lertworasirikul, A.; Noguchi, K.; Ogawa K. \& Okuyama, K. (2004). Plausible molecular and crystal structures of chitosan/HI type II salt. Carbohydrate Research, Vol.339, No.4, (March 2004), pp. 835-843. ISSN: 0008-6215.

Letendre, M.; D'Aprano, G.; Lacroix, M.; Salmieri, S. \& St-Gelais, D. (2002). Physicochemical properties and bacterial resistance of biodegradable milk protein films containing agar and pectin. Agricultural and Food Chemistry, Vol.50, No.21, (October 2002), pp. 6017-6022. ISSN: 0021-8561.

Lieberman, E. R. \& Gilbert, S. G. (1973). Gas Permeation of Collagen Films as Affected by Cross- linkage, Moisture, and Plasticizer Content. Journal of Polymer Science, Symposium, Vol.41, No.1, (1973), pp.33-43. ISSN: 1936-0959.

Liu, H. ; Du, Y.-M. \& John, F. K. (2007). Hydration energy of the 1,4-bonds of chitosan and their breakdown by ultrasonic treatment. Carbohydrate Polymers, Vol.68, No.3, (April 2007), pp.598-600. ISSN: 0144-8617. 
Liu, Z.; Li, X.; Peng, Z.; Tong, B. \& Liu, G. (1999). The mechanism of agglomeration and control in the process of ultrafine powder prepared by wet chemical method. Chemistry, Vol.7, No. 7, (August, 1999), pp. 54-57. ISSN: 0441-3776

Lizarraga, M. S.; De Piante Vicin, D.; Gonzalez, R.; Rubiolo, A. \& Santiago, L. G. (2006). Rheological behaviour of whey protein concentrate and k-carrageenan aqueous mixtures. Food Hydrocolloids, Vol.20, No.5, (July 2006), pp. 740-748. ISSN: 0268-005X.

Lourdin, D.; Della Valle, G. \& Colonna, P. (1995). Influence of amylose content on starch films and foams. Carbohydrate Polymer, Vol.27, No.4, (December 1995), pp. 261-270. ISSN: 0144-8617.

Lu, Y.; Weng, L. \& Cao, X. (2006). Morphological, thermal and mechanical properties of ramie crystallites reinforced plasticized starch biocomposites. Carbohydrate Polymers, Vol.63, No.1, (January 2006), pp. 1-7. ISSN: 0144-8617.

Manners, D. J. (1989). Recent developments in our understanding of amylopectin structure. Carbohydrate Polymers, Vol.11, No.2, (1989), pp. 87-112. ISSN 0144-8617.

Manoi, K. \& Rizvi, S. S. H. (2010). Physicochemical characteristics of phosphorylated crosslinked starch produced by reactive supercritical fluid extrusion. Carbohydrate Polymers, Vol.81, No.4, (July 2004), pp. 687-694. ISSN 0144-8617.

Masclaux, C.; Gouanve, F. \& Espuche, E. (2010). Experimental and modelling studies of transport in starch nanocomposite films as affected by relative humidity. Membrane Science, Vol.363, No.1-2, (November 2010), pp. 221-231. ISSN: 0376-7388.

McPherson, A. E. \& Jane, J. (1999). Comparison of waxy potato with other root and tuber starches Carbohydrate Polymers, Vol.40, No.1, (September 1999), pp.57-70. ISSN 01448617.

Ming, L.; Penf, L.; Wei, Z.; Long, Y.; Fengwei, X.; Huanyin, P.; Hongshen, L. \& Ling, C. (2011). Extrusion processing and characterization of edible starch films with different amylose contents. Food Engineering, Vol.106, No.1, (September 2011), pp. 95-101. ISSN: 0260-8774.

Morrison, W. R. (1995). Starch lipids and how they relate to starch granule structure and functionality. Cereal Foods World, Vol.40, No.6 (November 1995), pp. 437-446. ISSN: 0146-6283

Muller, C. M. O. ; Laurindo, J. B. \& Yamashita, F. (2011). Effect of nanoclay incorporation method on mechanical and water vapor barrier properties of starch-based films. Industrial Crops and Products, Vol.33, No.3, (May 2011), pp. 605-610. ISSN: 0926-6690.

Muzzarelli, R. A. A.; Biagini, G.; Mattioli-Belmonte, M.; Talassi, O.; Gandolfi, M. G.; Solmi, R.; Carraro, S.; Giardino, R.; Fini, M. \& Nicoli-Aldini, N. (1997). Osteoinduction by chitosan-complexed BMP. Morpho-structural response in an osteoporotic model. Bioactive and Compatible Polymers, Vol.12, No.2, (October 1997), pp. 321-329. ISSN: 15308030.

Olivier, A. ; Cazaux, F. ; Gors, C. \& Coqueret, X. (2001). Compatibilization of StarchAllylurea Blends by Electron Beam Irradiation: Spectroscopic Monitoring and Assessment of Grafting Efficiency. Biomacromolecules, Vol.2, No.4, (September 2001), pp. 1260-1266. ISSN:1526-4602. 
Pareta, R. \& Edirisinghe, M. J. (2006). A novel method for the preparation of biodegradable microspheres for protein drug delivery. Carbohydrate Polymers, Vol.63, No.3, (March 2006), pp. 425-431. ISSN: 0144-8617.

Park, S. Y.; Marsh, K. S. \& Rhim, J. W. (2002). Characteristics of different molecular weight chitosan films affected by the type of organic solvents. Food Sciences, Vol.67, No.1, (January 2002), pp. 194-197. ISSN: 1750-3841.

Peroval, C.; Debeaufort, F.; Despre, D. \& Voilley, A. (2002). Edible arabinoxylan-based films. I. Effects of lipids types on water vapor permeability, film structure and other physical characteristics. Agricultural and Food Chemistry, Vol.50, No.14, (July 2002), pp. 3977-3983. ISSN: 1520-5118.

Phan T. D.; Debeaufort ,F.; Voilley. \& A., Luu, D. (2009). Biopolymer interactions affect the functional properties of edible films based on agar, cassava starch and arabinoxylan blends. Food Engineering, Vol.90, No.4, (February 2009), pp. 548-558. ISSN: 0260-8774.

Phattaraporn, T.; Waranyou, S.; Fazilah, A. \& Thawien, W. (2010). Characteristics and properties of rice starch films reinforced with palm pressed fibers. International Food Research Journal, Vol.17, No.3, (2010), pp. 535-547. ISSN: 22317546.

Phattaraporn, T., Waranyou, S. \& Thawien, W. 2011. Effect of palm pressed fiber (PPF) surface treatment on the properties of rice starch films. International Food Research Journal. Vol.18, No. 1 (January 2011), pp. 535-547. ISSN: 22317546.

Podsiadlo, P.; Paternel, S.; Rouillard, J. M.; Zhang, Z.; Lee, J.; Lee, J. W.; Gulari, E. \& Kotov, N. A. (2005). Layer-by-layer assembly of nacre-like nanostructured composites with antimicrobial properties. Langmuir, Vol.21, No.25, (December 2005), pp.11915-11921. ISSN :1520-5827.

Rhim, W. J. \& Shellhammer, H. T. (2005). Lipid based edible films and coating. Innovations in food packaging, Academic Press, ISBN: 978-0-12-311632-1.

Rhim, J. W. (2004). Physical and chemical properties of water resistant sodium alginate films. Lebensmittel-Wissenschaft Und-Technologie, Vol.37, No.3, (May 2004), pp. 323-330. ISSN: 0023-6438.

Rindlav- Westling, A.; Stading, M. \& Gatenholm, P. (2002). Crystallinity and morphology in films of starch, amylase and amylopectin blends. Biomacromolecules, Vol.3, No.1, (January 2002), pp. 84-91.ISSN: 1525-7797.

Rindlav- Westling, A.; Stading, M.; Hermansson A. M. \& Gatenholm, P. (1998). Structure, mechanical and barrier properties of amylose and amylopectin films. Carbohydrate. Polymer, Vol.36, No.2-3, (July 1998), pp. 217-224. ISSN: 0144-8617.

Robeson, L. M. (2008). The upper bound revisited. Membrane Science, Vol.320, No.1-2, (July 2008), pp. 390-400. ISSN: 0376-7388.

Rutledge, J. E.; Islam, M. N. \& James, W. H. (1974). Improved canning stability of parboiled rice through cross-linking Cereal Chemistry, Vol.51, No.1, (January-February 1974), pp. 46-50. ISSN: 0009-0352.

Sabato, S.; Ouattara, B.; Yu, H.; D'Aprano, G.; Le Tien, C. \& Mateescu, M. (2001). Mechanical and barrier properties of cross-linked soy and whey protein based films. Agricultural and Food Chemistry. Vol.49, No.3, (March 2001), pp. 1397-1403.ISSN: 0021-8561. 
Shih, Y. F. (2007). Mechanical and thermal properties of waste water bamboo husk fiber reinforced epoxy composites. Material Science and Engineering Part A, Vol. 445-446, (February 2007), pp. 289-295. ISSN: 0921-5093.

Shogren, R. L. ; Lawton, J. W.; Tiefenbacher, K. F. \& Chen, L. (1998). Starch poly(vinyl alcohol) Foamed Articles Prepared by Baking Process. Applied Polymer Science, Vol.68, No.13, (June 1998), pp. 2129-2140. ISSN: 1097-4628.

Singh, J.; Kaur, L. \& McCarthy, O. J. (2007). Factors influencing the physico-chemical, morphological, thermal and rheological properties of some chemically modified starches for food applications-A review. Food Hydrocolloids, Vol.21, No.1, (January 2007), pp. 1-22. ISSN:0268-005X.

Smiley, R. A. \& Jackson, H. L. 2002. Chemistry and the Chemical Industry: A practical guide for non-chemists, CRC press, ISBN: 1-58716-054-4, Florida, USA.

Spielmann, H.; Lovell, W. W.; Holzle, E.; Johnson, B. E.; Maurer, T.; Miranda, M. A.; Pape, W. J. W.; Sapora, O. \& Sladowski, D. (1994). In Vitro Phototoxicity testing. The report and recommendations of ECVAM workshop 2. ATLA. Alternative to laboratory animals, Vol.22, No.5, pp.314-348. ISSN: 0261-1929.

Srinivasa, P. C.; Ramesh, M. N. \& Tharanathan, R. N. (2007). Effect of plasticizers and fatty acids on mechanical and permeability characteristics of chitosan films. Food Hydrocolloids, Vol.21, No.7, (October 2007), pp. 1113-1122. ISSN: 0268-005X.

Stading, M.; Rindlav-Westling, A. \& Gatenholm P. (2001). Humidity-induced Structural Transitions in Amylose and Amylopectin Films, Carbohydrate Polymers, vol.45, No.3, (July 2001), pp.209-217. ISSN: 0144-8617.

Weiss, J.; Takhistov, P. \& McClements, D. J. (2006). Functional materials in food nanotechnology. Food Science, Vol.71, No.9, (November/December 2006), pp. R107-R116. ISSN: 1750-3841.

Wittaya, T. (2009). Microcomposites of rice starch film reinforced with microcrystalline cellulose from palm pressed fiber. International Food Research Journal, Vol.16, No.4, (2009), pp. 493-500. ISSN: 22317546.

Woods, R. J. \& Pikaev, A. K. (1993). Applied Radiation Chemistry: Radiation Processing. Wiley, ISBN: 978-0-471-54452-4. New York

Wu, Y.; Geng, F.; Chang, P. R.; Yu, J. \& Ma, X. (2009). Effect of agar on the microstructure and performance of potato starch film. Carbohydrate Polymers, Vol.76, No.2, (March 2009), pp. 299-304. ISSN: 0144-8617.

Xu, X.Y.; Kim, K.M.; Hanna, M.A. \& Nag, D. (2005). Chitosan-starch composite film: preparation and characterization. Industrial Crops and Products an International Journal, Vol.21, No.2, (March 2005), pp.185-192. ISSN: 0926-6690.

Yang, L. \& Paulson, A. T. (2000). Effects of lipids on mechanical and moisture barrier properties of edible gellan film. Food Research International, Vol.33, No.7, (August 2000), pp. 571-578. ISSN: 0963-9969.

Yew, G. H.; Mohd Yusof, A. M.; Mohd Ishak, U.S. \& Ishiaku , Z.A. (2005). Water absorption and enzymatic degradation of poly(lactic acid)/rice starch composites. Polymer Degradation and Stability, Vol.90, No.3, (December 2005), pp. 488-500. ISSN: 0141-3910. 
134 Structure and Function of Food Engineering

Zhai, M.; Zhao,L.; Yoshii, F. \& Kume, T. (2004). Study on antibacterial starch/chitosan blend film formed under the action of irradiation. Carbohydrate Polymers, Vol.57, No.1, (August 2004), pp. 83-88. ISSN: 0144-8617. 